\title{
A Novel Mode-Matching Formulation of Multifurcated Waveguide Junctions
}

\author{
Guilherme S. Rosa ${ }^{1}$ (D) \\ ${ }^{1}$ Center for Telecommunications Studies, Department of Electrical Engineering, \\ Pontifical Catholic University of Rio de Janeiro, Rio de Janeiro 22451-900, Brazil, \\ guilhermeSimonDaRosa@cetuc.puc-rio.br
}

\begin{abstract}
In this paper, we present a mode-matching-based formulation for the electromagnetic analysis of multifurcated waveguide problems, that is, the junction of a number of input waveguides with an output region of larger cross-section area. A generalized scattering matrix (GSM) representation is obtained for relating the forward and backward modal field amplitudes in each of the waveguides in terms of coupling integrals representing the conservation of the reaction of the electromagnetic fields. Numerical results for several multifurcated coaxial waveguide devices are provided to validate the formulation. Comparisons against the finite-element method demonstrate that the present approach can accurately model multifurcated waveguide problems. The method introduced here provides useful matrix formulas that allow us to model multi-port waveguide devices by reusing well-known coupling integrals of two-port problems.
\end{abstract}

Index Terms - Generalized scattering matrix, mode-matching technique, multifurcated waveguide

\section{INTRODUCTION}

The mode-matching technique (MMT) is an efficient method frequently employed in the designing of microwave devices, such as filters, impedance-matching transformers, power dividers, and couplers. Besides today many computational electromagnetic methods are able to properly solve such problems, the MMT excels in the modeling of geometries conforming with Cartesian and cylindrical coordinates [1]-[5], [6, Chs. 9 and 10] because when combined with the generalized scattering matrix (GSM) it renders a very efficient framework for the rigorous design of complex wave-guided circuits [4], [7].

Over the years, many works have used mode-matching-based formulations for obtaining a GSM representing the junction between two waveguides with different cross-sectional areas. This kind of boundary condition problem usually is solved by enforcing the conservation of the reaction [8] or the conservation of the complex power [9] across the waveguide step discontinuity. Closed-form coupling integrals are well-known in the literature for modeling the junction between rectangular, circular, coaxial, elliptical, among other common waveguides [2], [6], [10]-[14].

In this paper, we will discuss the modeling of multi-port devices via the MMT. In the past, bifurcated and trifurcated junctions in parallel waveguides were considered in [15], [16] via the Wiener-Hopf and the residue-calculus techniques. More generic multi-port discontinuities were also studied in [17] in the context of parallel-plate guided structures. The multifurcation of rectangular waveguides where analyzed in [18], and the importance of higher-order mode interactions were demonstrated by experimental results. Other related cases of multi-port junctions were analyzed in [19] and in [11] for the coupling 
problems between cylindrical and elliptical waveguides, respectively. In these works, the GSM that represents an $N$-furcated problem (as illustrated in Fig. 1) was handled by the superposition of the GSMs for the step discontinuities between regions 1 and $N, 2$ and $N, 3$ and $N$, etc. The simultaneous coupling is then incorporated into a unified GSM relating all the $N-1$ input waveguide regions with the output region $N$. We present an alternative approach for obtaining the GSM that describes the overall problem by avoiding the intermediate calculation of the GSMs of $N-1$ step-junction discontinuities. Our formulation relates the coupling integrals directly with the unified scattering matrix. The present method can be seen as a generalization of the MMT solution in [20], where particular bifurcated waveguide junctions were analyzed, namely, geometries with two input waveguides connected to one output waveguide. In the present notation, this corresponds to a total of $N=3$ waveguides. The introduction of novel matrix formulas herein has allowed obtaining GSMs in a more polished and general form for an arbitrary number of waveguides $N$. In addition, our novel MMT formulation offers an inherent physical interpretation in terms of reflection and transmission parcels that allow us to make a comprehensive description of the modal coupling phenomena in terms of the forward and backward modal amplitudes of one waveguide with the other coupled regions. The present matrix formulation also allows a straightforward model to waveguide terminations by just updating some sub-matrices of the GSM formalism, without requiring the redefinition of eigenfunction in such cavity-backed scenarios. This characteristic confers an advantage upon the method in [20] and others found in the specialized MMT literature [4], [11], [18], [19]. In short, the major contribution of this work is the formulation of a matrix-friendly GSM representation of multifurcated waveguide junctions that can recycle the coupling integrals of two-port devices that are already known in the MMT literature.

The rest of this paper is organized as follows. In Section II we first present a mathematical description of fields in each guided region, and we then enforce the conservation of the reaction across the coupling plane. A GSM is obtained for relating the forward and backward modal field amplitudes in each of the waveguides. We also show a simple procedure for modeling the effects of one short-circuited waveguide by updating the GSM derived in the non-truncated multifurcated problem. Section III presents a series of numerical results to illustrate the ability of the present formulation to correct modeling multifurcated problems. We have selected the junction between coaxial waveguides in the numerical investigations, each one excited by the transverse electromagnetic (TEM) mode. We explore the rising of high-order modes (such as the transverse magnetic mode $\mathrm{TM}_{01}$ ) in the larger waveguide and its influence in the reflection experienced at the input ports. Finally, Section IV provides some concluding remarks. 


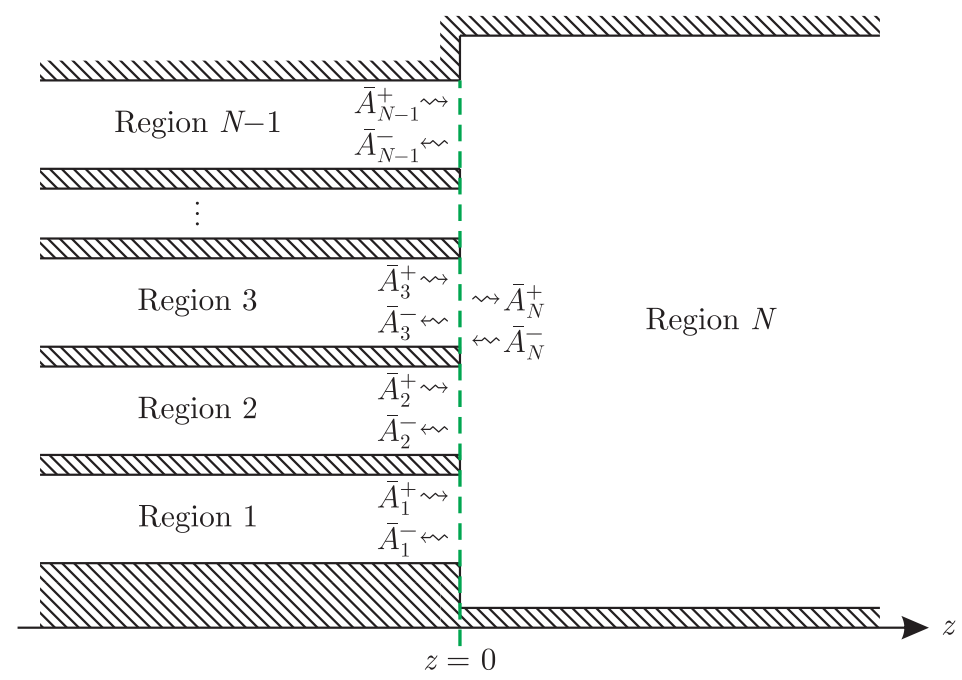

Fig. 1. Geometry of the multifurcated waveguide. The PEC regions are represented by the hatched areas.

\section{THEORETICAL Formulation}

In what follows, we employ a notation similar to that in [21], [22], where the time-harmonic dependence in the form $\exp (-i \omega t)$ is assumed and suppressed. Let us consider the multifurcated waveguide problem depicted in Fig. 1, where a set of $N-1$ input regions are coupled with the output region $N$. Each waveguide is semi-infinite along the longitudinal direction, and they are transversely truncated by a perfect electric conductor (PEC). The cross-sectional area of regions $j=\{1,2, \ldots, N\}$ are denoted by $S_{j}$, and we assume the field coupling at $z=0$ is over $S_{M}$, with $M=\{1,2, \ldots, N-1\}$. We also assume that $S_{M}$ is contained into $S_{N}$, i.e., $S_{M} \in S_{N}$.

The electromagnetic field is expressed in each region as a sum of appropriated modal parcels, and at the junction $z=0$, we must enforce the continuity of the electric and magnetic fields transversal to $z$ according to

$$
\sum_{m=1}^{\infty}\left(A_{j, m}^{+}+A_{j, m}^{-}\right) \mathbf{e}_{s j, m}(\rho, \phi)=\sum_{n=1}^{\infty}\left(A_{N, n}^{+}+A_{N, n}^{-}\right) \mathbf{e}_{s N, n}(\rho, \phi)
$$

and

$$
\sum_{m=1}^{\infty}\left(A_{j, m}^{+}-A_{j, m}^{-}\right) \mathbf{h}_{s j, m}(\rho, \phi)=\sum_{n=1}^{\infty}\left(A_{N, n}^{+}-A_{N, n}^{-}\right) \mathbf{h}_{s N, n}(\rho, \phi)
$$

inside $S_{j}, j=\{1,2,3, \ldots, N-1\}$. In the above, $\mathbf{e}_{s j, m}(\rho, \phi)$ and $\mathbf{h}_{s j, m}(\rho, \phi)$ are the $m$ th electric and magnetic field components transversal to $z$ in region $j$, respectively, and they were expressed as a sum of forward $\left(A_{j, m}^{+}\right)$and backward $\left(A_{j, m}^{-}\right)$field amplitudes with respect to the coupling plane $z=0$. The fields are represented in cylindrical coordinates $(\rho, \phi, z)$ for convenience, and the subscript $s$ is used herein to indicate components transversal to $z$. By imposing the conservation of the reaction [8], [22], [23] of the fields across the coupling apertures $S_{N}-S_{j}, j=1,2, \ldots, N-1$, at $z=0$, (1) and (2) give rise to the matrix equations

$$
\sum_{j=1}^{N-1} \overline{\bar{X}}_{j, N}\left(\bar{A}_{j}^{+}+\bar{A}_{j}^{-}\right)=\overline{\bar{Q}}_{N}\left(\bar{A}_{N}^{+}+\bar{A}_{N}^{-}\right)
$$


and

$$
\overline{\bar{X}}_{j, N}^{T}\left(\bar{A}_{j}^{+}-\bar{A}_{j}^{-}\right)=\overline{\bar{Q}}_{j}\left(\bar{A}_{N}^{+}-\bar{A}_{N}^{-}\right), \text {for } j=\{1,2,3, \ldots, N-1\},
$$

where the superscript $T$ indicates the transpose of the matrix, and the forward and backward amplitudes of the fields in region $j$ were assembled as

$$
\bar{A}_{j}^{ \pm}=\left[\begin{array}{c}
A_{j, 1}^{ \pm} \\
A_{j, 2}^{ \pm} \\
A_{j, 3}^{ \pm} \\
\vdots
\end{array}\right],
$$

and the entries of matrices $\overline{\bar{X}}_{j, N}$ and $\overline{\bar{Q}}_{j}$ are related with the reaction and self-reaction integrals according to

$$
\begin{aligned}
\left.\overline{\bar{X}}_{i, j}\right|_{m, n} & =\int_{S_{i}}\left[\mathbf{e}_{s i, n}(\rho, \phi) \times \mathbf{h}_{s j, n}(\rho, \phi)\right] \cdot \hat{z} d s, \\
\left.\overline{\bar{Q}}_{j}\right|_{m, n} & =\left.\delta_{m, n} \overline{\bar{X}}_{j, j}\right|_{m, n},
\end{aligned}
$$

where $\hat{z}$ denotes the unit vector pointing to $+z$, and $\delta_{m, n}$ denotes the Kronecker delta function (equal to 1 if $m=n$, and 0 otherwise) and its presence is due to the modal orthogonality over the waveguide cross-section. The coupling integrals that yields $\overline{\bar{X}}_{j, i}$ are calculated over the aperture between regions $j$ and $i$, i.e., over $S_{j}$, with $j=1,2, \ldots, N-1$. In (3) and (4) we only need the reaction integrals $\overline{\bar{X}}_{j, i}$ between the fields in regions $j=1,2, \ldots, N-1$ to region $i=N$. In contrast, the self-reaction integrals $\overline{\bar{Q}}_{j}$ are calculated over the cross-section area $S_{j}$ of all the regions $j=1,2, \ldots, N$.

In order to simplify the notation, let us introduce the matrices

$$
\begin{gathered}
\overline{\overline{\mathcal{X}}}_{M, N}=\left[\begin{array}{lllll}
\overline{\bar{X}}_{1, N} & \overline{\bar{X}}_{2, N} & \overline{\bar{X}}_{3, N} & \ldots & \overline{\bar{X}}_{N-1, N}
\end{array}\right], \\
\overline{\overline{\mathcal{Q}}}_{M}=\operatorname{diag}\left(\overline{\bar{Q}}_{1}, \overline{\bar{Q}}_{2}, \overline{\bar{Q}}_{3}, \ldots, \overline{\bar{Q}}_{N-1}\right),
\end{gathered}
$$

and the vectors

$$
\overline{\mathcal{A}}_{M}^{ \pm}=\left[\begin{array}{c}
\bar{A}_{1}^{ \pm} \\
\bar{A}_{2}^{ \pm} \\
\bar{A}_{3}^{ \pm} \\
\vdots \\
\bar{A}_{N-1}^{ \pm}
\end{array}\right],
$$

such as the boundary conditions in (3) and (4) can be written in a compact manner as

$$
\begin{aligned}
& \overline{\overline{\mathcal{X}}}_{M, N}\left(\overline{\mathcal{A}}_{M}^{+}+\overline{\mathcal{A}}_{M}^{-}\right)=\overline{\bar{Q}}_{N}\left(\bar{A}_{N}^{+}+\bar{A}_{N}^{-}\right), \\
& \overline{\overline{\mathcal{X}}}_{M, N}^{T}\left(\bar{A}_{N}^{+}-\bar{A}_{N}^{-}\right)=\overline{\overline{\mathcal{Q}}}_{M}\left(\overline{\mathcal{A}}_{M}^{+}-\overline{\mathcal{A}}_{M}^{-}\right) .
\end{aligned}
$$

To proceed, let us isolate $\bar{A}_{N}^{+}$in (11) and substitute it in (12) to obtain

$$
\overline{\mathcal{A}}_{M}^{-}=\left[\overline{\overline{\mathcal{X}}}_{M, N}^{T} \overline{\bar{Q}}_{N}^{-1} \overline{\overline{\mathcal{X}}}_{M, N}+\overline{\overline{\mathcal{Q}}}_{M}\right]^{-1}\left\{\left[\overline{\overline{\mathcal{Q}}}_{M}-\overline{\overline{\mathcal{X}}}_{M, N}^{T} \overline{\bar{Q}}_{N}^{-1} \overline{\overline{\mathcal{X}}}_{M, N}\right] \overline{\mathcal{A}}_{M}^{+}+2 \overline{\overline{\mathcal{X}}}_{M, N}^{T} \bar{A}_{N}^{-}\right\},
$$

where the superscript -1 indicates the inverse of the matrix. Similarly, by isolating $\bar{A}_{N}^{+}$in (12) and 
substituting it in (11) yields

$$
\bar{A}_{N}^{+}=\left[\overline{\overline{\mathcal{X}}}_{M, N} \overline{\overline{\mathcal{Q}}}_{M}^{-1} \overline{\overline{\mathcal{X}}}_{M, N}^{T}+\overline{\bar{Q}}_{N}\right]^{-1}\left\{\left[\overline{\overline{\mathcal{X}}}_{M, N} \overline{\overline{\mathcal{Q}}}_{M}^{-1} \overline{\overline{\mathcal{X}}}_{M, N}^{T}-\overline{\bar{Q}}_{N}\right] \bar{A}_{N}^{-}+2 \overline{\overline{\mathcal{X}}}_{M, N} \overline{\mathcal{A}}_{M}^{+}\right\} .
$$

\section{A. Generalized Scattering Matrix}

From (13) and (14), we can relate the forward and backward modal amplitudes in each waveguide as

$$
\left[\begin{array}{c}
\overline{\mathcal{A}}_{M}^{-} \\
\bar{A}_{N}^{+}
\end{array}\right]=\overline{\bar{S}}\left[\begin{array}{c}
\overline{\mathcal{A}}_{M}^{+} \\
\bar{A}_{N}^{-}
\end{array}\right],
$$

where the GSM is given by

$$
\overline{\bar{S}}=\left[\begin{array}{ll}
\overline{\bar{R}}_{M, N} & \overline{\bar{T}}_{N, M} \\
\overline{\bar{T}}_{M, N} & \overline{\bar{R}}_{N, M}
\end{array}\right],
$$

and comprises

$$
\begin{aligned}
\overline{\bar{R}}_{M, N} & =-\overline{\bar{D}}_{M+}^{-1} \overline{\bar{D}}_{M-} \\
\overline{\bar{T}}_{N, M} & =2 \overline{\bar{D}}_{M+}^{-1} \overline{\overline{\mathcal{X}}}_{M, N}^{T} \\
\overline{\bar{T}}_{M, N} & =2 \overline{\bar{D}}_{N+}^{-1} \overline{\overline{\mathcal{X}}}_{M, N} \\
\overline{\bar{R}}_{N, M} & =\overline{\bar{D}}_{N+}^{-1} \overline{\bar{D}}_{N-},
\end{aligned}
$$

where

$$
\begin{aligned}
\overline{\bar{D}}_{M \pm} & =\overline{\overline{\mathcal{X}}}_{M, N}^{T} \overline{\bar{Q}}_{N}^{-1} \overline{\mathcal{X}}_{M, N} \pm \overline{\overline{\mathcal{Q}}}_{M} \\
\overline{\bar{D}}_{N \pm} & =\overline{\overline{\mathcal{X}}}_{M, N} \overline{\overline{\mathcal{Q}}}_{M}^{-1} \overline{\overline{\mathcal{X}}}_{M, N}^{T} \pm \overline{\bar{Q}}_{N}
\end{aligned}
$$

The GSM submatrices in (17) can also be expressed in their extended forms:

$$
\begin{aligned}
& \overline{\bar{R}}_{M, N}=\left[\begin{array}{cccc}
\overline{\bar{R}}_{1, N} & \overline{\bar{T}}_{2,1} & \cdots & \overline{\bar{T}}_{N-1,1} \\
\overline{\bar{T}}_{1,2} & \overline{\bar{R}}_{2, N} & \cdots & \overline{\bar{T}}_{N-1,2} \\
\vdots & \vdots & \ddots & \vdots \\
\overline{\bar{T}}_{1, N-1} & \overline{\bar{T}}_{2, N-1} & \cdots & \overline{\bar{R}}_{N-1, N-1}
\end{array}\right], \\
& \overline{\bar{T}}_{N, M}=\left[\begin{array}{c}
\overline{\bar{T}}_{N, 1} \\
\overline{\bar{T}}_{N, 2} \\
\vdots \\
\overline{\bar{T}}_{N, N-1}
\end{array}\right] \\
& \overline{\bar{T}}_{M, N}=\left[\begin{array}{llll}
\overline{\bar{T}}_{1, N} & \overline{\bar{T}}_{2, N} & \cdots & \overline{\bar{T}}_{N-1, N}
\end{array}\right] .
\end{aligned}
$$

In the above, $\overline{\bar{R}}_{i, j}$ and $\overline{\bar{T}}_{i, j}$ can be physically interpreted as the reflection and transmission matrices, respectively, of the modal amplitudes from region $i$ to region $j$. For example, in view of (15) and (16), the backward modal field amplitudes in region 1 can be mathematically written as

$$
\bar{A}_{1}^{-}=\overline{\bar{R}}_{1, N} \bar{A}_{1}^{+}+\overline{\bar{T}}_{2,1} \bar{A}_{2}^{+}+\overline{\bar{T}}_{3,1} \bar{A}_{3}^{+}+\cdots+\overline{\bar{T}}_{N-1,1} \bar{A}_{N-1}^{+}+\overline{\bar{T}}_{N, 1} \bar{A}_{N}^{-} .
$$

A physical representation of the above construction is depicted in Fig. 2. 


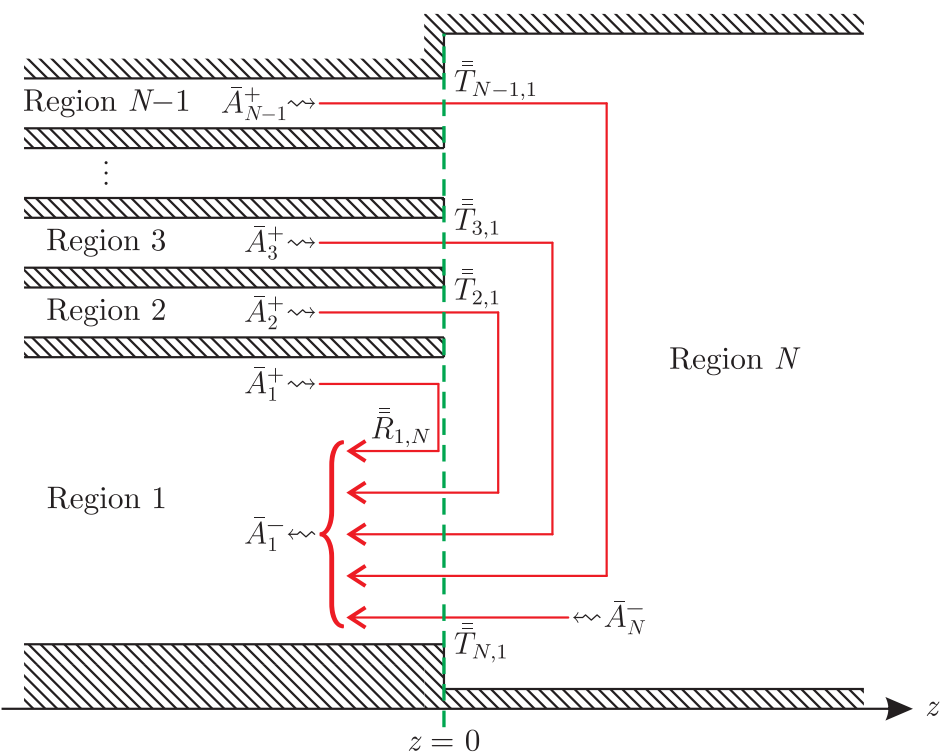

Fig. 2. Physical representation of the backward modal field amplitudes in region 1.

\section{B. Normalization}

For practical applications, it is important to express the GSM matrix in a way that it becomes independent of the excitation field magnitude. A normalization of the modal fields $\mathbf{e}_{s j, m}(\rho, \phi)$ and $\mathbf{h}_{s j, m}(\rho, \phi)$ in (1) and (2) can be chosen to ensure a unitary power over the cross-section $S_{j}$ at $z=0$ for each of the modes. Consequently, we can express the normalized GSM matrix $\hat{\overline{\bar{S}}}$ as

$$
\begin{aligned}
& \hat{\overline{\bar{R}}}_{M, N}=\operatorname{abs}\left(\overline{\overline{\mathcal{Q}}}_{M}\right)^{1 / 2} \overline{\bar{R}}_{M, N} \operatorname{abs}\left(\overline{\overline{\mathcal{Q}}}_{M}\right)^{-1 / 2} \\
& \hat{\overline{\bar{T}}}_{N, M}=\operatorname{abs}\left(\overline{\overline{\mathcal{Q}}}_{M}\right)^{1 / 2} \overline{\bar{T}}_{N, M} \operatorname{abs}\left(\overline{\bar{Q}}_{N}\right)^{-1 / 2} \\
& \hat{\overline{\bar{T}}}_{M, N}=\operatorname{abs}\left(\overline{\bar{Q}}_{N}\right)^{1 / 2} \overline{\bar{T}}_{M, N} \operatorname{abs}\left(\overline{\overline{\mathcal{Q}}}_{M}\right)^{-1 / 2} \\
& \hat{\overline{\bar{R}}}_{N, M}=\operatorname{abs}\left(\overline{\bar{Q}}_{N}\right)^{1 / 2} \overline{\bar{R}}_{N, M} \operatorname{abs}\left(\overline{\bar{Q}}_{N}\right)^{-1 / 2},
\end{aligned}
$$

in which we should update the former GSM by pre- and post-multiplying it by the square root of the modulus of the diagonal-defined self-reaction matrices $\overline{\bar{Q}}_{j}$ and its inverse, respectively. The above normalization provides the properties $\operatorname{det}(\hat{\overline{\bar{S}}})=+1$ and $\hat{\overline{\bar{S}}} \hat{\overline{\bar{S}}}=\overline{\bar{I}}$, where $\overline{\bar{I}}$ is an identity matrix. For further details, see [23].

\section{Waveguide Termination}

We consider now a special case where the region $j$ is not an excitation port, but at $z=-d_{j}$ this waveguide is characterized by a reflection matrix $\overline{\bar{R}}_{j, 0}$, as depicted in Fig. 3. Such reflection matrix can represent a junction of other waveguides as well as a short-circuited plate, and in general, we must enforce the condition $\bar{A}_{j}^{-}=\overline{\bar{P}}_{j} \overline{\bar{R}}_{j, 0} \overline{\bar{P}}_{j} \bar{A}_{j}^{+}$, where $\overline{\bar{P}}_{j}$ is a diagonal matrix defined by $\left.\overline{\bar{P}}_{j}\right|_{n, n}=$ $\exp \left(i k_{j z, n} d_{j}\right)$, where $k_{j z, n}$ is the axial wavenumber of the $n$th mode in region $j$. Consequently, we can obtain new scattering coefficients independent of $\bar{A}_{j}^{ \pm}$. In region $r=1, r \neq j$, for example, we can relate the forward and backward modal field amplitudes via a generalized reflection matrix given by

$$
\tilde{\overline{\bar{R}}}_{1, N}=\overline{\bar{R}}_{1, N}-\overline{\bar{T}}_{j, 1}\left(\overline{\bar{R}}_{j, N}-\overline{\bar{P}}_{j} \overline{\bar{R}}_{j, 0} \overline{\bar{P}}_{j}\right)^{-1} \overline{\bar{T}}_{1, j}
$$


If the waveguide is truncated by a PEC, we can simplify the above by using $\overline{\bar{R}}_{j, 0}=-\overline{\bar{I}}$. Expressions similar to (25) can be obtained for all of the remaining reflection and transmission matrices. In the case of other truncations in regions $j+1, j+2$, etc., we can systematically eliminate the amplitudes $\bar{A}_{j+1}^{ \pm}, \bar{A}_{j+2}^{ \pm}$, etc., and then obtain the corresponding generalized $\overline{\bar{R}}$ and $\overline{\bar{T}}$ matrices.

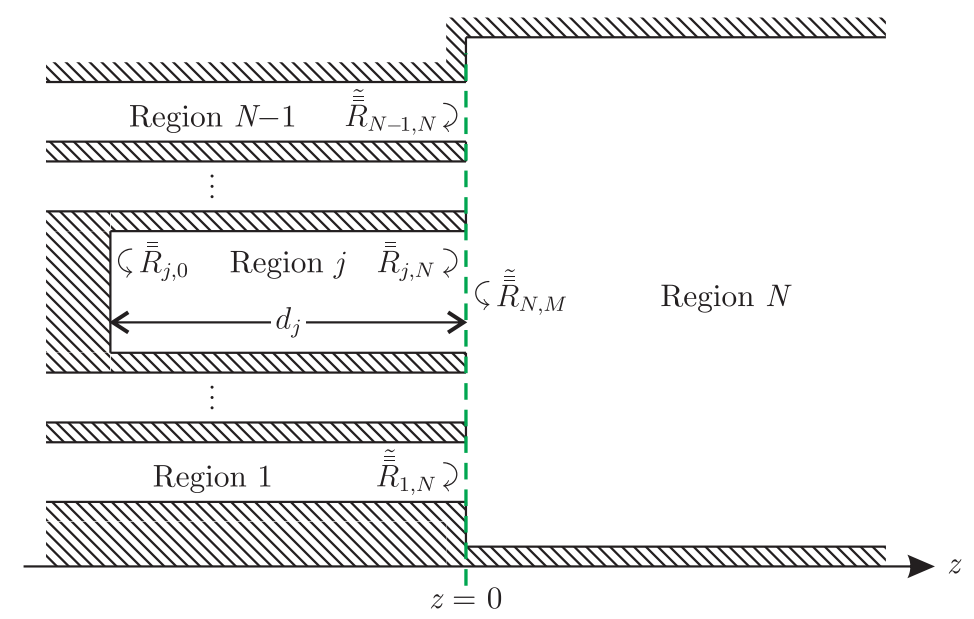

Fig. 3. Geometry of the multifurcated waveguide where region $j$ is truncated.

\section{NUMERICAL RESULTS AND DisCUSSIONS}

To illustrate the validity of the above-presented formulation, we consider a generic multifurcated geometry with a number of coaxial waveguide inputs (regions 1,2,3, and 4) connected to an output coaxial waveguide (region 5). These waveguides are defined by the parameters presented in Table I. Notice the number of modal fields employed to describe each waveguide region was chosen properly to avoiding relative convergence issues [24]. Also, all waveguides are filled with vacuum and are radially truncated by PECs. The mode-matching coupling integrals we have used to computing the reaction integrals (6) and (7) are available in closed-forms in [14], [20], [22], [25], [26] and will not be repeated here. In what follows, we will compare the results of the present approach with those obtained by a frequency-domain finite-element method (FEM) from CST [27]. We investigated the reflection coefficient of the TEM modes in each waveguide as a function of the frequency, and in order to assess the effect of high-order modes, we have selected the frequency range of $10 \mathrm{GHz}-50 \mathrm{GHz}$.

TABLE I. Simulation parameters.

\begin{tabular}{cccc}
\hline Region & Inner radius $(\mathrm{mm})$ & Outer radius $(\mathrm{mm})$ & Number of modes \\
\hline \hline 1 & 1.0 & 2.0 & 4 \\
2 & 2.5 & 3.5 & 4 \\
3 & 4.0 & 5.0 & 4 \\
4 & 5.5 & 6.0 & 4 \\
5 & 1.0 & 6.0 & 16 \\
\hline
\end{tabular}

\section{A. Waveguide with Step Discontinuity}

As a first example, we consider an ordinary junction between two coaxial waveguides characterized by the parameters of regions 1 and 5 (the larger waveguide) shown in Fig. 4. In this particular case, $\overline{\overline{\mathcal{Q}}}_{M}$ 
in (9) becomes equal to the self-reaction matrix $\overline{\bar{Q}}_{1}$, while $\overline{\overline{\mathcal{X}}}_{M, N}$ in (8) reduces to the coupling-reaction matrix $\overline{\bar{X}}_{1,5}$. Accordingly, the GSM components in (17) turn equivalent to those presented in [10, eq. 6] 1 .

Fig. 5 shows the reflection coefficient in decibel for the TEM mode in regions 1 and 5 as a function of the frequency. Good agreement is observed between the presented MMT formulation and the FEM results. The cut-off frequency of the $\mathrm{TM}_{01}$ mode in region 5 is at $28.96 \mathrm{GHz}$, and we can observe that the propagation of this modal field affects the reflection coefficient of the TEM mode in regions 1 and 5. Up to this cut-off frequency, the reflection response in waveguides 1 and 5 are exactly the same because of only TEM modes are propagating fields. Above $28.96 \mathrm{GHz}$, however, the TEM mode in region $1\left(\mathrm{TEM}^{(1)}\right)$ couples a parcel of its fields with the TEM mode in region $5\left(\mathrm{TEM}^{(5)}\right)$ and a parcel with the $\mathrm{TM}_{01}$ mode in region $5\left(\mathrm{TM}_{01}^{(5)}\right)$. As a consequence, the reflection coefficient of $\mathrm{TEM}^{(1)}$ decreases at high frequencies, as observed in Fig. 5.

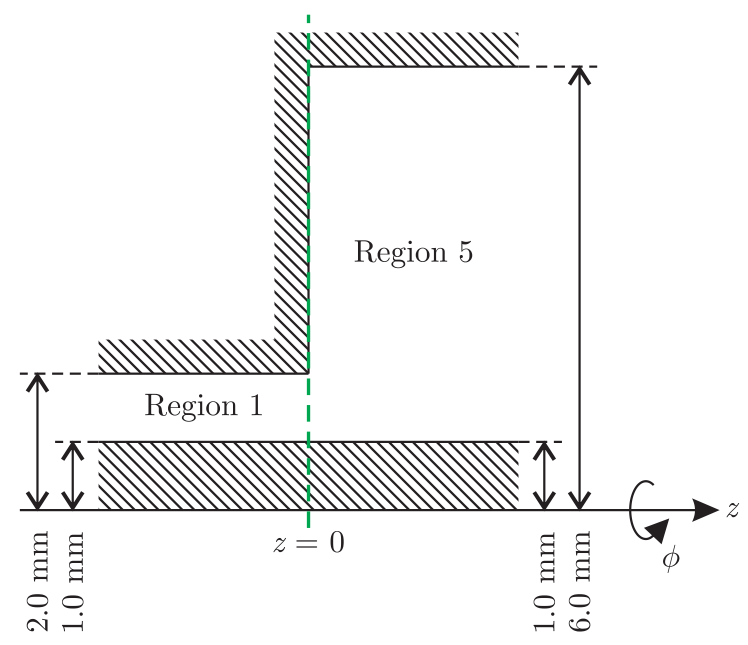

Fig. 4. Geometry of a waveguide with step discontinuity.

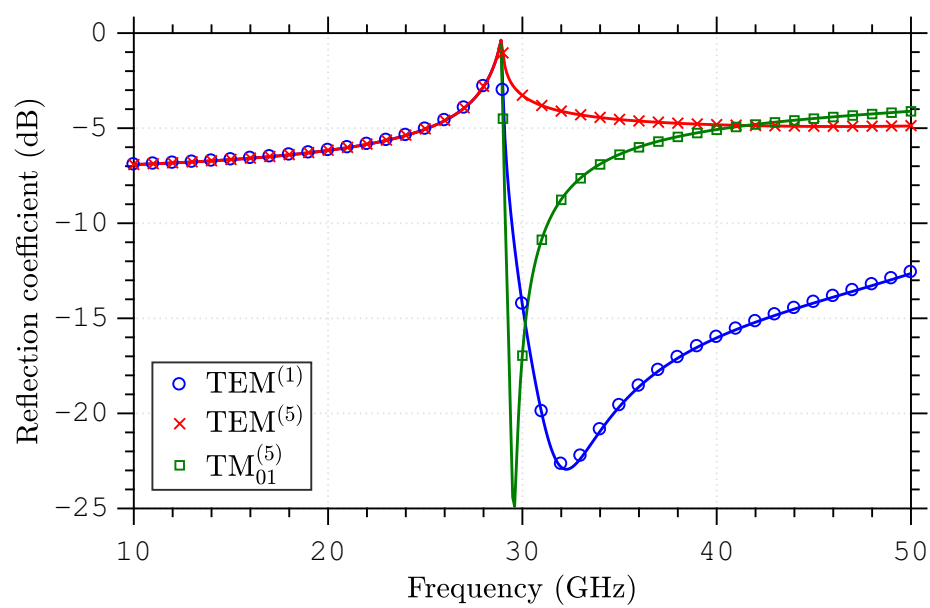

Fig. 5. Reflection coefficient in decibel for the TEM mode in regions 1 and 5 , and for the $\mathrm{TM}_{01}$ mode in region 5 as a function of the frequency. The results from the present algorithm are indicated by the solid lines while the small symbols are FEM results from [27].

\footnotetext{
${ }^{1}$ Notice the notation presented here is different from that in [10], but equivalence is obtained by substituting the reaction matrices in compliance with $\overline{\bar{Q}}_{1} \rightarrow \underline{\underline{R}}, \overline{\bar{Q}}_{5} \rightarrow \underline{\underline{Q}}$, and $\overline{\bar{X}}_{1,5} \rightarrow \underline{\underline{P}}$. 


\section{B. Bifurcated Waveguide}

We now consider a geometry where the latter problem received an additional coaxial waveguide (region 2), as depicted in Fig. 6. This bifurcated waveguide structure couples the input regions 1 and 2 with the output region 5. Fig. 7 shows the reflection coefficient in decibel for the mode TEM in regions 1, 2 and 5 as a function of the frequency. Good agreement is observed between the MMT and the FEM, and again we can recognize that the propagation of the $\mathrm{TM}_{01}$ mode in region 5 modifies significantly the reflection experienced by the TEM mode in regions 1 and 5 . In a minor degree, the reflection of the TEM mode in region 2 is also affected by the rising of the $\mathrm{TM}_{01}$ mode in region 5 .

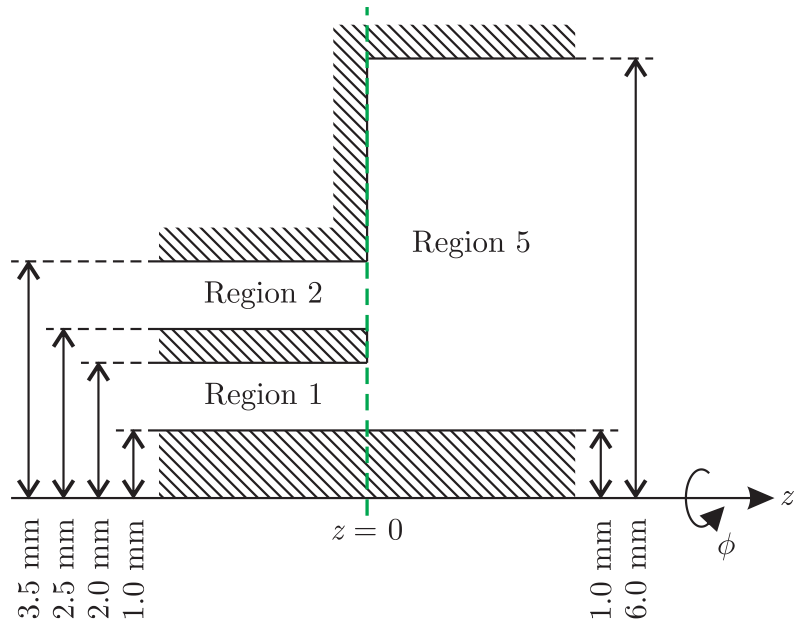

Fig. 6. Geometry of the bifurcated waveguide problem.

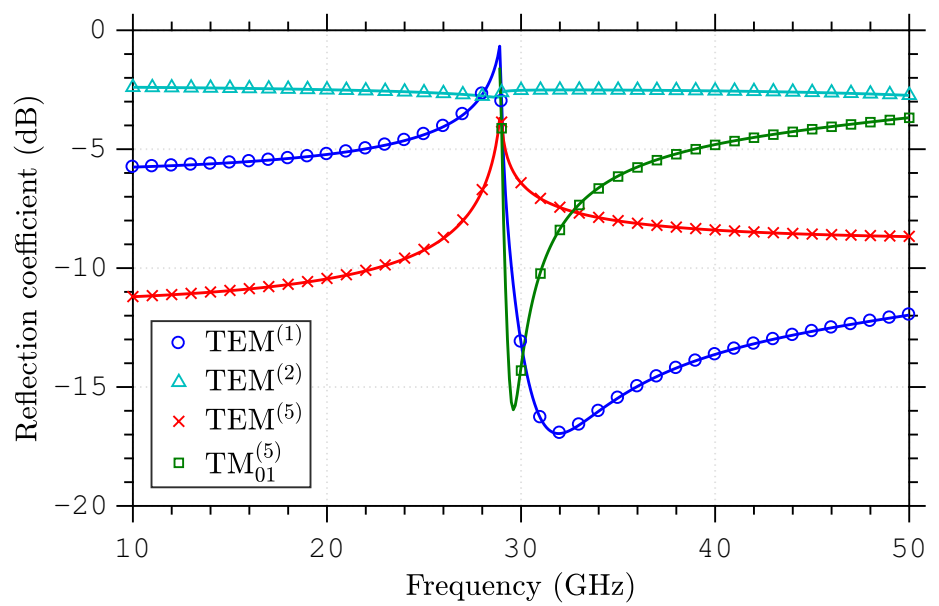

Fig. 7. Reflection coefficient in decibel for the TEM mode in regions 1,2 , and 5 , and for the $\mathrm{TM}_{01}$ mode in region 5 as a function of the frequency. The results from the present algorithm are indicated by the solid lines while the small symbols are FEM results from [27].

\section{Trifurcated Waveguide}

By the inclusion of another coaxial waveguide (region 3), we obtain a trifurcated geometry that couples the input regions 1, 2, and 3 with the output region 5, as shown in Fig. 8. As before, the simulation parameters are shown in Table I. Fig. 9 shows the reflection coefficient in decibel for the mode TEM in regions $1,2,3$ and 5 , and for $\mathrm{TM}_{01}$ mode in region 5 . The results obtained from the presented MMT formulation again shown good agreement with those of the FEM. 


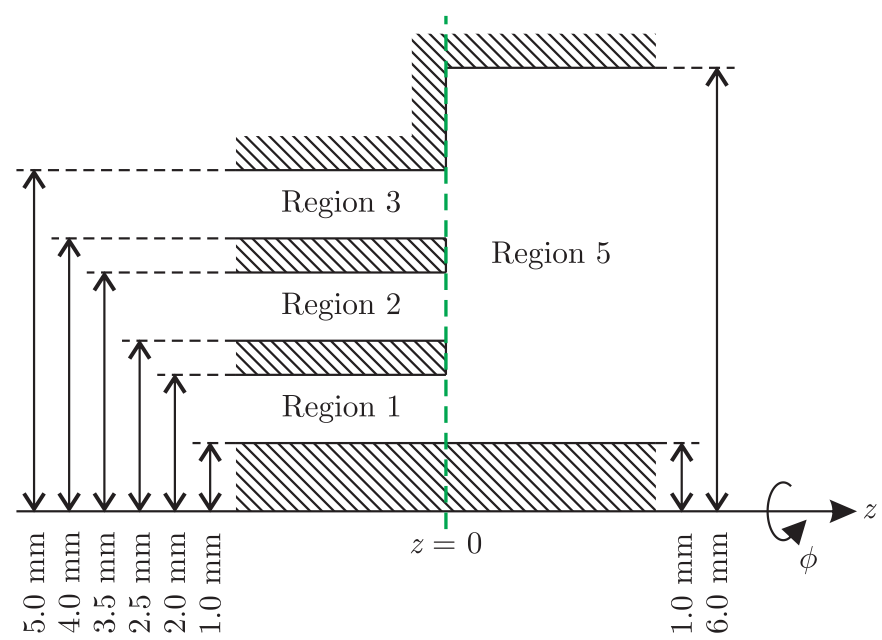

Fig. 8. Geometry of the trifurcated waveguide problem.

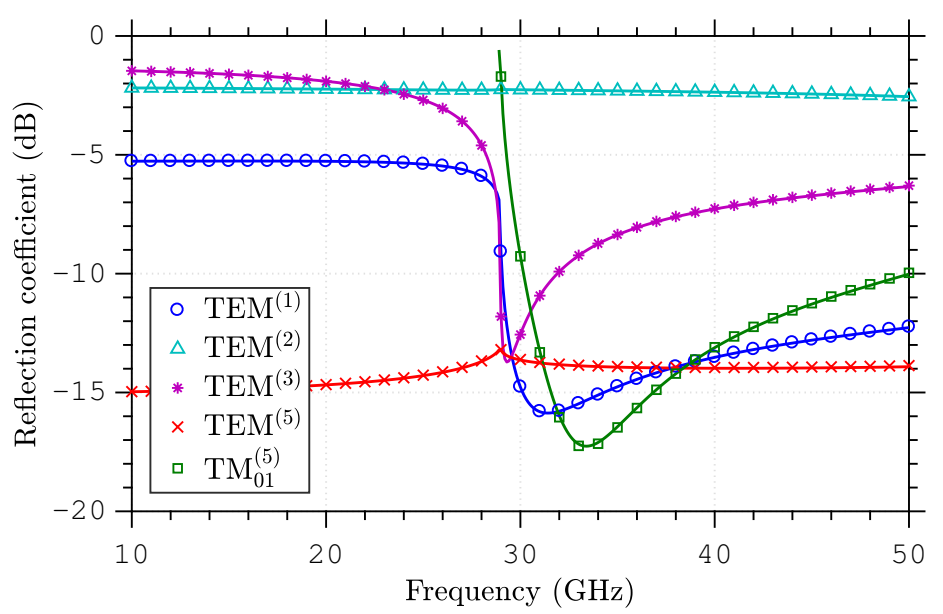

Fig. 9. Reflection coefficient in decibel for the TEM mode in regions $1,2,3$, and 5 , and for the $\mathrm{TM}_{01}$ mode in region 5 as a function of the frequency. The results from the present algorithm are indicated by the solid lines while the small symbols are FEM results from [27].

\section{Quadfurcated Waveguide}

We consider now a geometry that couples four inputs waveguides, i.e., regions 1, 2, 3, and 4, with the output region 5, as depicted in Fig. 10. Fig. 11 shows the reflection coefficient in decibel for the TEM mode in each region and the $\mathrm{TM}_{01}$ mode in region 5 as a function of the frequency. The results obtained via the presented formulation are again in agreement with those of the FEM.

\section{E. Waveguide with Step Discontinuity and with a Radial Slot}

Finally, we consider the geometry presented in Fig. 12 to verify the predictions of (25). The problem consists of the coupling between the input regions 1 and 2 with the output region 5 , similar to bifurcation analyzed above in Section III-B but with a PEC truncating region 2 at $z=-d_{2}$. Figures 13 and 14 plot the reflection coefficient in decibel for the TEM mode in regions 1 and 5 as a function of the frequency and four different values of $d_{2}: 0 \mathrm{~mm}, 2 \mathrm{~mm}, 4 \mathrm{~mm}$, and $6 \mathrm{~mm}$. Very good agreement is observed versus the FEM results from [27]. 


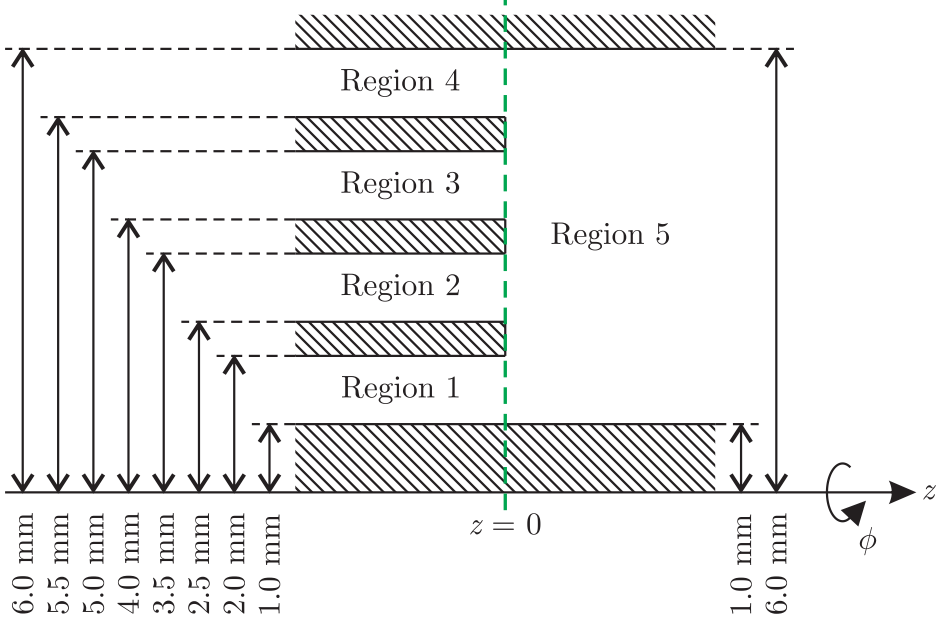

Fig. 10. Geometry of the quadfurcated waveguide problem.

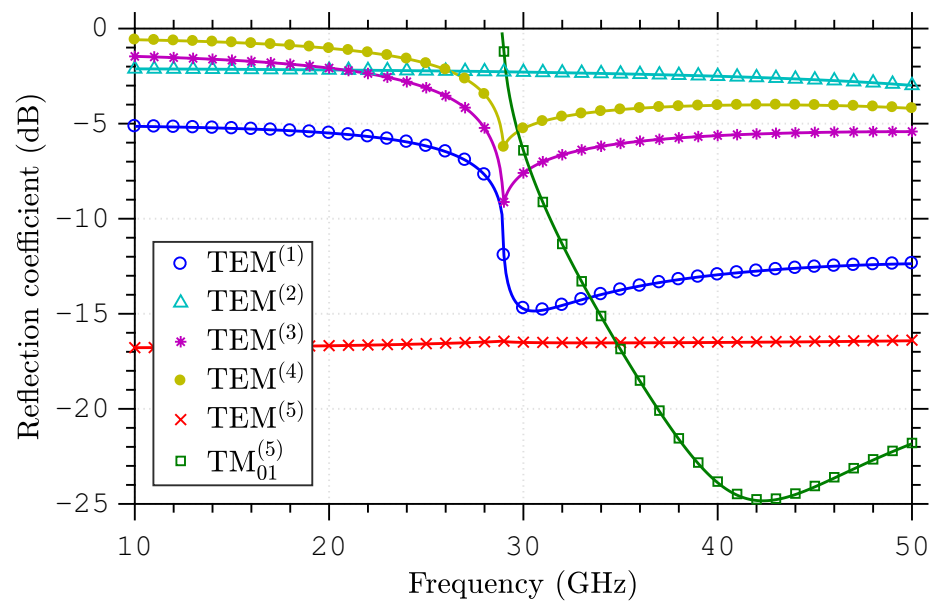

Fig. 11. Reflection coefficient in decibel for the TEM mode in regions $1,2,3$, 4 , and 5 , and for the $\mathrm{TM}_{01}$ mode in region 5 as a function of the frequency. The results from the present algorithm are indicated by the solid lines while the small symbols are FEM results from [27].

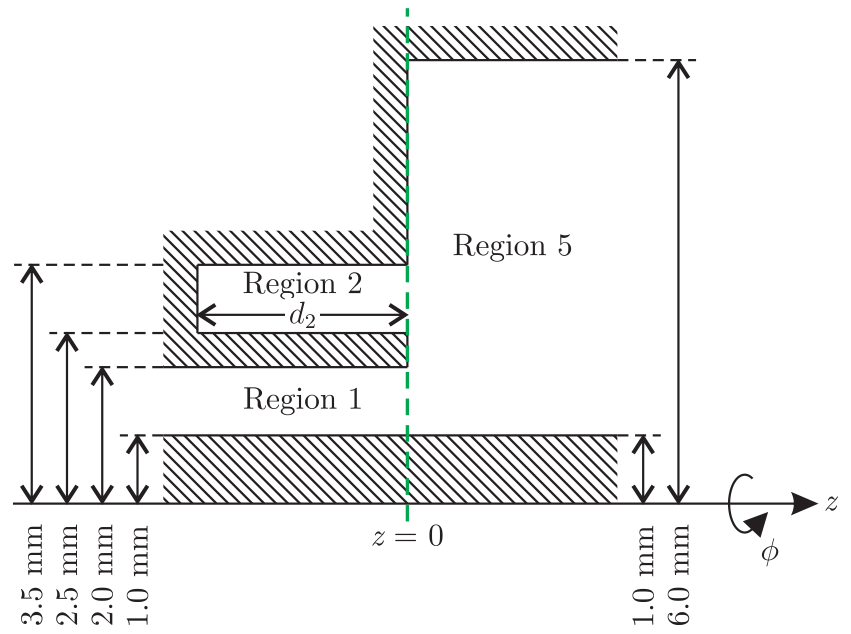

Fig. 12. Geometry of the bifurcated waveguide problem with a radial slot. 
Journal of Microwaves, Optoelectronics and Electromagnetic Applications, Vol. 20, No. 3, September 2021 DOI: http://dx.doi.org/10.1590/2179-10742021v20i31292

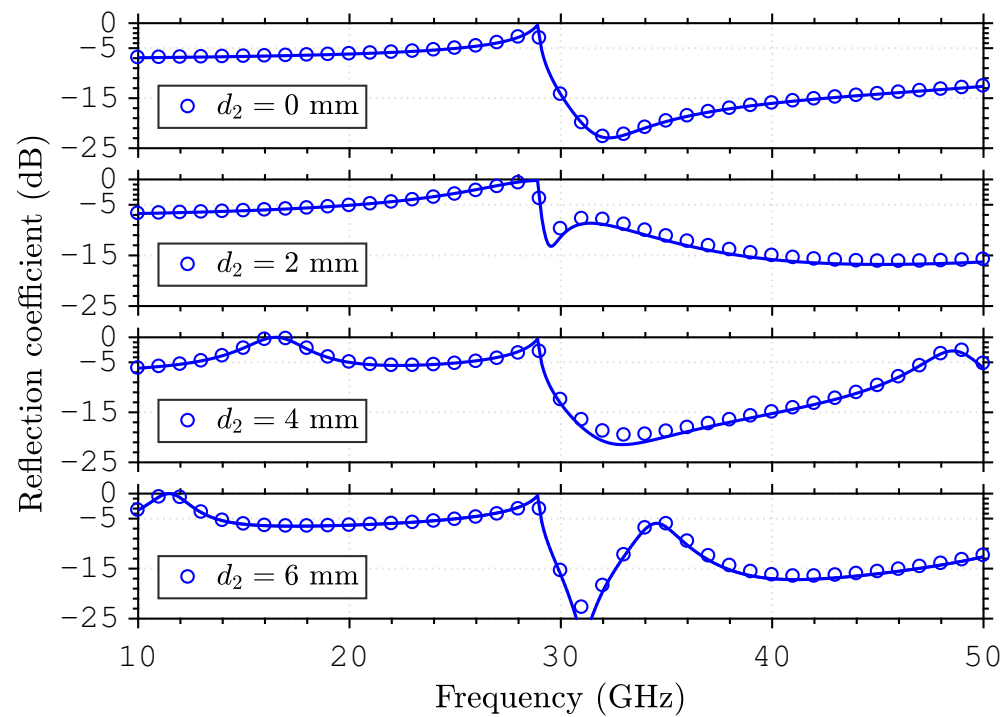

Fig. 13. Reflection coefficient in decibel for the TEM mode in region 1 as a function of the frequency for a short-circuit placed in region 2 at $z=-d_{2}$. The results from the present algorithm are indicated by the solid lines while the small dots are FEM results from [27].

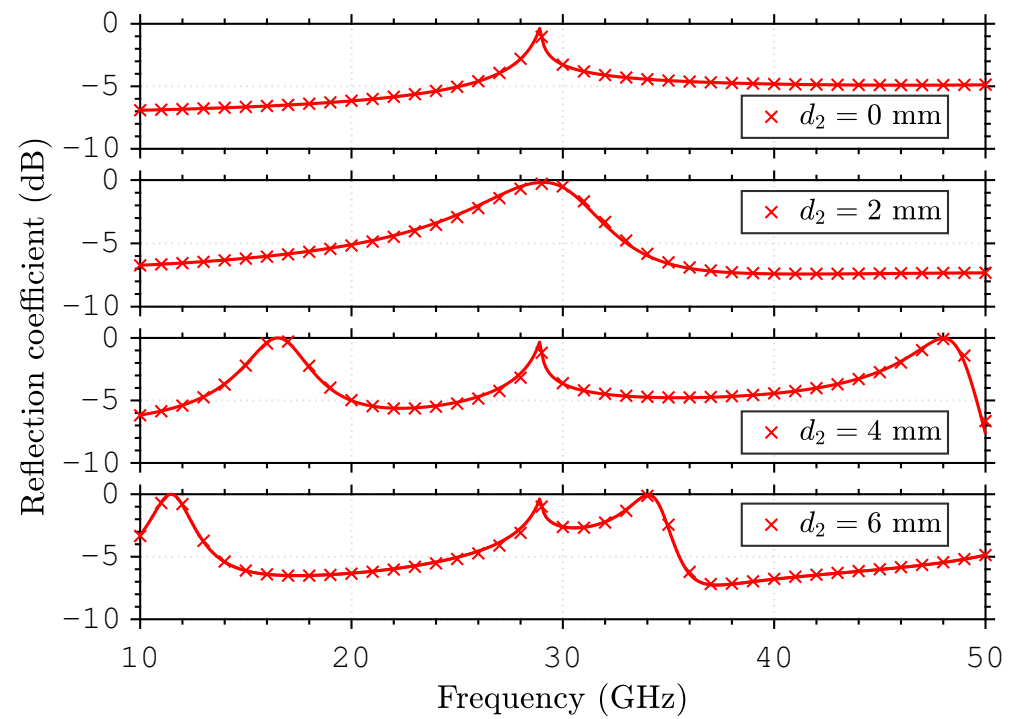

Fig. 14. Reflection coefficient in decibel for the TEM mode in region 5 as a function of the frequency for a short-circuit placed in region 2 at $z=-d_{2}$. The results from the present algorithm are indicated by the solid lines while the small crosses are FEM results from [27]. 


\section{CONCLUSION}

A mode-matching formulation of multifurcated waveguide junctions was explored in this paper. Formulas for obtaining a normalized GSM were presented in terms of reaction matrices. We have also shown a procedure for incorporating the effects of a short-circuited waveguide into the derived GSM. An illustrative geometry comprising several coupled coaxial waveguides was used to validate our approach. The numerical results show that the present method can accurately model such multifurcated waveguide problems.

The analysis of multifurcated waveguide junctions can also be effectively handled using spacediscretization methods such as finite-differences, finite-elements and finite-volumes. The high computational effort demanded by these methods, however, contrasts in many order of magnitude in terms of CPU time and RAM requirements when compared to MMT-based solutions. In addition, the accuracy of full-wave MMT analyses where proved in many works when closed-form eigenfunction expansions are used for standard waveguides. For further details, please see the comprehensive list of MMT works organized in [4]. In special, as a consequence of the employment of closed-form coupling integrals for the geometries analyzed in this work, it is important to notice the computational cost of our method is very low when compared with that of the FEM. The procedure presented here is not restricted to axially symmetric geometries and can be useful for the modeling of multi-port devices by reusing closed-form results of many well-known MMT formulations of waveguide step discontinuities.

\section{ACKNOWLEDGMENTS}

This work was supported in part by the Brazilian Agency CAPES under Grant \#001 and in part by Brazilian Agency CNPq under Grant \#426378/2018-0.

\section{REFERENCES}

[1] A. Wexler, "Solution of waveguide discontinuities by modal analysis," IEEE Trans. Microw. Theory Techn., vol. 15, no. 9, pp. 508-517, Sep. 1967.

[2] R. Mittra and S. W. Lee, Analytical techniques in the theory of guided waves. Macmillan, 1971.

[3] W. J. English, "The circular waveguide step-discontinuity mode transducer (short papers)," IEEE Trans. Microw. Theory Techn., vol. 21, no. 10, pp. 633-636, Oct. 1973.

[4] F. Arndt, R. Beyer, J. M. Reiter, T. Sieverding, and T. Wolf, "Automated design of waveguide components using hybrid mode-matching/numerical em building-blocks in optimization-oriented cad frameworks-state of the art and recent advances,” IEEE Trans. Microw. Theory Techn., vol. 45, no. 5, pp. 747-760, May 1997.

[5] J. A. Ruiz-Cruz, J. R. Montejo-Garai, and J. M. Rebollar, "Computer aided design of waveguide devices by modematching methods," in Passive Microwave Components and Antennas, V. Zhurbenko, Ed. Rijeka: IntechOpen, 2010, ch. 6 .

[6] T. Itoh, Numerical Techniques for Microwave and Millimeter-Wave Passive Structures. Wiley, 1989.

[7] J. M. Reiter and F. Arndt, "Rigorous analysis of arbitrarily shaped h- and e-plane discontinuities in rectangular waveguides by a full-wave boundary contour mode-matching method," IEEE Trans. Microw. Theory Techn., vol. 43, no. 4, pp. 796801, Apr. 1995.

[8] V. H. Rumsey, "Reaction concept in electromagnetic theory," Phys. Rev., vol. 94, pp. 1483-1491, Jun. 1954.

[9] J. D. Wade and R. H. MacPhie, "Conservation of complex power technique for waveguide junctions with finite wall conductivity," IEEE Trans. Microw. Theory Techn., vol. 38, no. 4, pp. 373-378, Apr. 1990.

[10] G. L. James, "Analysis and design of $\mathrm{TE}_{11}$-to-HE $\mathrm{HE}_{11}$ corrugated cylindrical waveguide mode converters," IEEE Trans. Microw. Theory Techn., vol. 29, no. 10, pp. 1059-1066, Oct. 1981.

[11] M. Mongiardo, P. Russer, C. Tomassoni, and L. B. Felsen, "Analysis of n-furcation in elliptical waveguides via the generalized network formulation,” IEEE Trans. Microw. Theory Techn., vol. 47, no. 12, pp. 2473-2478, Dec. 1999. 
Journal of Microwaves, Optoelectronics and Electromagnetic Applications, Vol. 20, No. 3, September 2021

DOI: http://dx.doi.org/10.1590/2179-10742021v20i31292

[12] L. C. Silva, "Determination of the scattering matrix of ring-loaded corrugated waveguide mode converters," IEEE Trans. Microw. Theory Techn., vol. 37, no. 3, pp. 646-649, Mar. 1989.

[13] Z. Shen and R. H. MacPhie, "Scattering by a thick off-centered circular iris in circular waveguide," IEEE Trans. Microw. Theory Techn., vol. 43, no. 11, pp. 2639-2642, Nov. 1995.

[14] A. P. Orfanidis, G. A. Kyriacou, and J. N. Sahalos, "A mode-matching technique for the study of circular and coaxial waveguide discontinuities based on closed-form coupling integrals," IEEE Trans. Microw. Theory Techn., vol. 48, no. 5, pp. 880-883, May 2000.

[15] G. F. VanBlaricum and R. Mittra, "A modified residue-calculus technique for solving a class of boundary value problems part i: Waveguide discontinuities," IEEE Trans. Microw. Theory Techn., vol. 17, no. 6, pp. 302-309, Jun. 1969.

[16] J. R. Pace and R. Mittra, “The trifurcated waveguide,” Radio Science, vol. 1, no. 1, pp. 117-122, Jan. 1966.

[17] R. R. Mansour and R. H. Macphie, "Scattering at an n-furcated parallel-plate waveguide junction (short paper)," IEEE Trans. Microw. Theory Techn., vol. 33, no. 9, pp. 830-835, Sep. 1985.

[18] J. Esteban and J. M. Rebollar, "Generalized scattering matrix of generalized two-port discontinuities: application to four-port and nonsymmetric six-port couplers," IEEE Trans. Microw. Theory Techn., vol. 39, no. 10, pp. 1725-1734, Oct. 1991.

[19] F. Dai, "Scattering and transmission matrix representations of multiguide junctions," IEEE Trans. Microw. Theory Techn., vol. 40, no. 7, pp. 1538-1544, Jul. 1992.

[20] A. L. dos Santos Lima, G. Simon da Rosa, and J. R. Bergmann, "A mode-matching solution for the study of cylindrical waveguide bifurcation via closed-form coupling integrals," AEU - International Journal of Electronics and Communications, vol. 118, p. 153135, 2020.

[21] W. C. Chew, Waves and Fields in Inhomogeneous Media. New York, NY, USA: John Wiley \& Sons, 1995.

[22] G. S. Rosa, J. R. Bergmann, and F. L. Teixeira, "A robust mode-matching algorithm for the analysis of triaxial welllogging tools in anisotropic geophysical formations," IEEE Trans. Geosci. Remote Sens., vol. 55, no. 5, pp. 2534-2545, May 2017.

[23] G. V. Eleftheriades, A. S. Omar, L. P. B. Katehi, and G. M. Rebeiz, "Some important properties of waveguide junction generalized scattering matrices in the context of the mode matching technique," IEEE Trans. Microw. Theory Techn., vol. 42, no. 10, pp. 1896-1903, Oct. 1994.

[24] M. Leroy, "On the convergence of numerical results in modal analysis," IEEE Trans. Antennas Propag., vol. 31, no. 4, pp. 655-659, Jul. 1983.

[25] G. S. Rosa, "Electromagnetics wave propagation in coaxial guides with non-homogeneous load excited by the TEM Mode.” Master's thesis, Dept. Elect. Eng., Pontifical Catholic University of Rio de Janeiro, Rio de Janeiro, RJ, Brazil, 2013, in Portuguese, DOI: 10.17771/PUCRio.acad.35451.

[26] _ - "Pseudo-analytical modeling for electromagnetic well-logging tools in complex geophysical formations," Ph.D. dissertation, Dept. Elect. Eng., Pontifical Catholic University of Rio de Janeiro, Rio de Janeiro, RJ, Brazil, 2017, DOI: 10.17771/PUCRio.acad.30559.

[27] CST AG, CST Studio Suite 2020, Darmstadt, Germany, 2020.

Brazilian Microwave and Optoelectronics Society-SBMO

Brazilian Society of Electromagnetism-SBMag received 7 Apr 2021; for review 12 Apr 2021; accepted 21 July 2021 (C) 2021 SBMO/SBMag 